\title{
Life Prediction of Thin Wall Pipe for External Superficial Cracked Exposed to Interior Pressure
}

\author{
${ }^{1} \mathrm{M}$. Abdulrazzaq and ${ }^{2}$ Mahmoud A. Hassan \\ ${ }^{1}$ Department of Materials Engineering, \\ ${ }^{2}$ Department of Mechanical Engineering, College of Engineering, University of Al-Qadisiyah, \\ Al-Diwaniyah, Iraq
}

\begin{abstract}
In this research, numerous thin pipes are exposed to complex cyclic loading range going, since, little vibration to huge capacity abrasions. This investigation offerings displaying of fatigue crack growth performance in thin partition of pipe for external superficial fractured pipe exposed to inside pressure. There are numerous variables influencing of fatigue crack growth, for example, crack length, condenses of the pipe and the R-ratio. Fatigue crack growth development as result of administration loads relies upon a wide range of contributing variables. With the assistance of a recreation fatigue crack growth in three-dimensional structures can either be anticipated or if nothing else be clarified for effectively existing failures. This study objective is to reproduce the influence of utilizing FCG Walker Model. The expected results had shown the Walker Model most applicable model for the VA loading. Simulation gave distinctive estimations of these variables which demonstrated their impacts on the fatigue crack growth development in this application. Furthermore, study need to consider alternate components towards robustness.
\end{abstract}

Key words: FCG, parametric angle, initial crack length, thin wall pipe, orientation, application

\section{INTRODUCTION}

Thin-walled pipes subjected to high interior pressure are approximately utilized as a part of the element plants, tig welding, and liquid stream with extremely basic controls of outline and service for safety. Hence with a specific end goal to guarantee wellbeing from startling fatigue failure $\mathrm{F}$ fand to expand the life of the thin wall pipe, Fatigue failure Ff investigation was awesome significance in proficient plan and facility (Ghidini and Donne, 2009; Bian and Lim, 2003). Fatigue crack FC by large shape and spread on the superficial of a thin-surface pipe exposed to cyclic inner pressure, where the most extreme tractable extraneous anxiety happens. The calculation of fatigue properties of structures to stay away from basic fatigue were perceived as engineering issues in the early many years of the 20 th century. The FCG model demonstrates "fits" the information appropriately, it might be utilized as a part of directing a FCG investigation. This estimation models for crack growth $\mathrm{CG}$ factor under variable loading VAL contrast from basic adjustment arranged the constant loading CAL benchmark active to multifaceted models with itemized portrayal of the important fracture mecanics FM. A few simulations, for example, ascertain the $\mathrm{CG}$ through averaging over the connected load range while numerous different simulations have tend to figure the $C G$ with cycle-by-cycle examination. Figure 1 demonstrate a schematic log-log plot rate of crack growth per cycle $(\mathrm{da} / \mathrm{dN})$ vs. crack length (a). FCGR curve indicating retardation following a solitary tensile over-load (Kocanda and Jasztal, 2012). For various materials, it has been surveyed that an initial increasing speed of FCG rate happens instantly after an over-load. The FCG rate at that point decelerates to a minimum value sooner or later in front of the over-load application and soon thereafter, the rate step by step comes back to its enduring state value (Huang et al., 2008; Kocanda and Jasztal, 2012; Stephens et al., 2000). The spectacle is otherwise called retardation. What's more, when different multiple tensile overloads are firmly connected they might cooperate with each other upgradeor can be reduced to retardation of general CG. Such over-load connection remains subject to the comparative size and the separating among the over-loads (Kocanda and Jasztal, 2012; Stephens et al., 2000) or the frequency of the overload application. FCG under irregular and VAL stacking have been dissected by a wide range of researchers like Chen and Lin (2008), Alaoui et al. (2009), Remes et al. (2017), Siqueira et al. (2010), Huang et al.

Corresponding Author: M. Abdulrazzaq, Department of Materials Engineering, College of Engineering, 


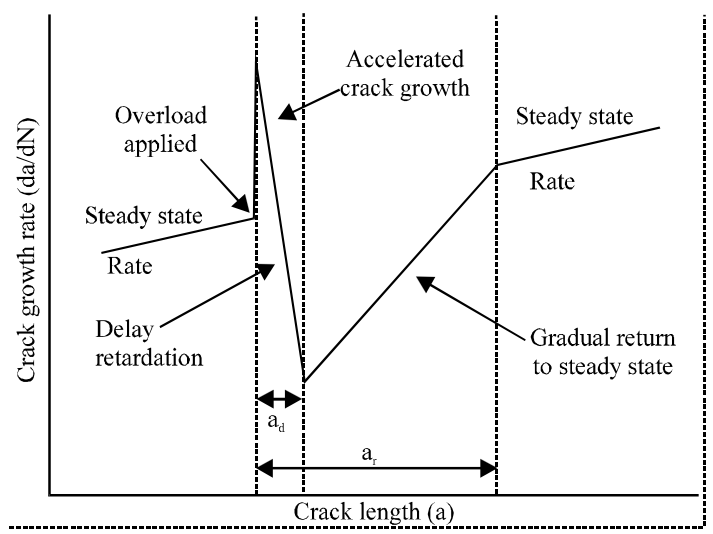

Fig. 1:Diagram CG rate curve performance delayed retardation following tensile over-load (Kocanda and Jasztal, 2012)

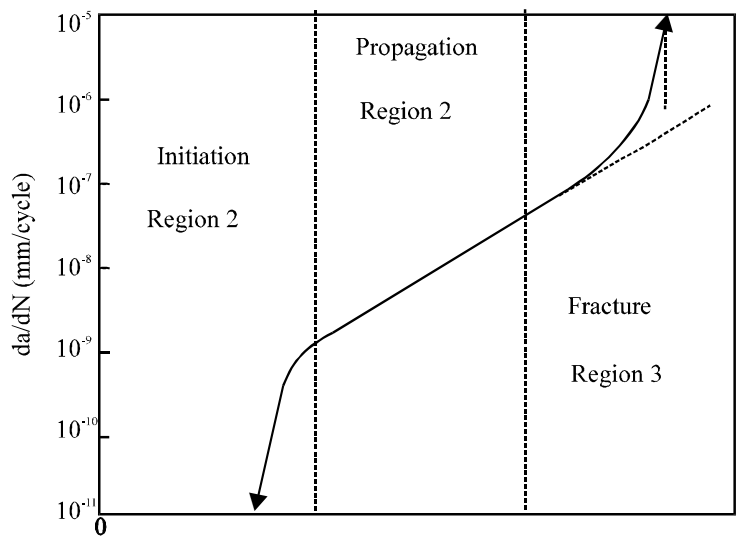

Fig. 2: Typical da/dN versus $\Delta \mathrm{K}$ curve

(2008), Kocanda and Jasztal (2012) and Stephens et al. (2000). Some of them give careful consideration to the simulation of delegate load histories of characterized irregular loading processes (Huang et al., 2008; Kocanda and Jasztal, 2012).

Figure 2 portrays a schematic plot of CGR per cycle $(\mathrm{da} / \mathrm{dn}) \mathrm{vs}$. Stress intensity factor range $(\Delta \mathrm{K})$ which illustrates a typical behaviour of FCG in metals. At the intermediate $\Delta \mathrm{K}$ values, we observe that the CGR curve in the region 1 and 3 deviates from the linear trend from low and high depend on $\Delta \mathrm{K}$ levels but the curve in the region 2 linear (propagation stage) (Siqueira et al., 2010).

Therefore, the overall Fatigue Life (FL) of a component is generally defined as follows: "Total life $=$ Initiation life+Propagation life". This can be characterized in a block diagram as in Fig. 3.

Literature review: It is difficult to classify a Fatigue Crack (FC) in the structures during service. It has been found

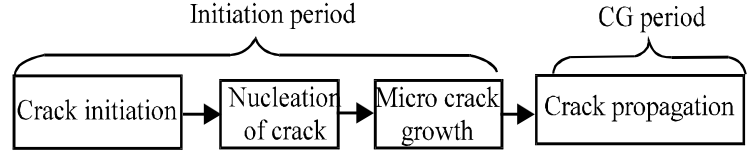

Fig. 3: Different phases of the fatigue life and relevant factors

from the literature review that no universal fatigue model has been developed to analyse the crack growth condition under the Variable Amplitude Load (VAL) and narrow range of applications. The characteristic of FCG behavior conduct was recognized before 1960 's, a particular collection of destruction model demonstrates for calculation of development lower than a wide assortment of administration loads has been deficient. The crack growth in constructions depends on the amplitude, frequency of the load and stress ratio (Huang et al., 2008). Due to the random nature of VAL, it is difficult to correctly model all these influential parameters. The purpose for construction prototypes is to interface hypothetical concepts with the experimental facts to give a decent expectation of future perceptions. Displaying of FCG data has upgraded the capability to make damage accepting outline methods of insight. The Walker modifies law verifiably models threshold and the beginning of fastcrack and is communicated in the accompanying calculation (Kocanda and Jasztal, 2011).

\section{MATERIALS AND METHODS}

Experimental work: Composite steel are generally utilized as a part of outline of many manufacturing application, AISI 302 stainless steel has a positive reaction to warm conduct (for the most part oil extinguishing took after by hardening) and a decent mix of flexibility and quality when treated along these lines and utilizing numerous application, for example cables, screws, springs and disc springs. In this application a thin pipe $(76.2 \mathrm{~mm}$ in span, $20 \mathrm{~mm}$ in diameter and $0.7 \mathrm{~mm}$ in thickness) through inside surface crack under loop stress has beeninvestigated. The material is chosen and appeared in Table 1. The load ranges account and mean of the utilized history of loading, rain flow method strategy was utilized. The most extreme tensile and pressure peck is $340 \mathrm{MPa}$, hence the stress ratio $(R=-1)$. The material utilized was a sort stainless steel AISI 302. The synthetic composition of the material (wt.\%) is recorded in Table 1. The simulation of the analysis was performed createdof (Zinecrack Software). This software to achieveby automatic crack mesh generation depends on fatigue and load condition. Dumbbell-shaped specimens with a 


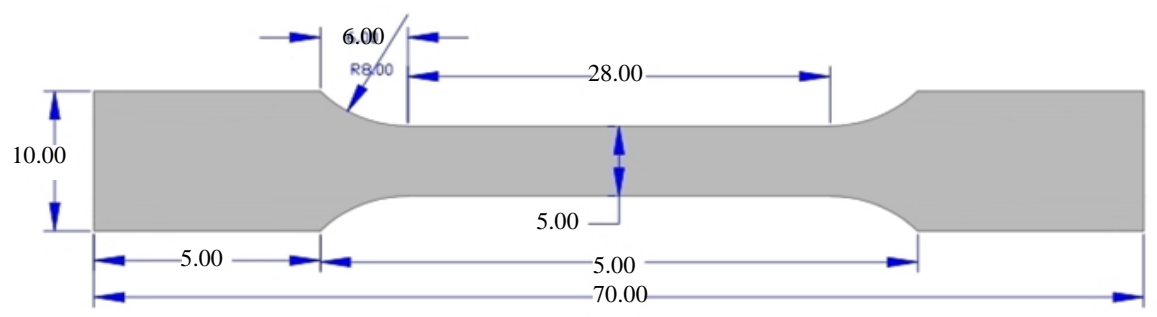

Fig. 4: Specimen configuration according to ASTM 313M

Table 1: Chemical composition (wt. \%)

\begin{tabular}{ll}
\hline Varriables & Values \\
\hline $\mathrm{C}$ & 0.08 \\
$\mathrm{Mn}$ & 2.00 \\
$\mathrm{~F}$ & 0.045 \\
$\mathrm{~S}$ & 0.03 \\
$\mathrm{Si}$ & 1.0 \\
$\mathrm{Cr}$ & $18.0-20.0$ \\
$\mathrm{Ni}$ & $8.0-11.0$ \\
\hline
\end{tabular}

Table 2: Mechanical and fatigue properties of AISI 302

\begin{tabular}{lr}
\hline Variables & Values \\
\hline Yield strength (MPa & 810 \\
Ultimate strength (MPa) & 1150 \\
Modulus of elasticity (GPa) & 180 \\
Hardness (Brinell) & 200 \\
Elongation at Break (\%) & 26.4 \\
\hline
\end{tabular}

Table 3: The material cyclic properties of AISI 302

\begin{tabular}{lr}
\hline Variables & Values \\
\hline Fatigue strength coefficient (MPa) & 920.000 \\
Fatigue strength exponent & -0.106 \\
Fatigue ductility exponent & -0.470 \\
Fatigue ductility coefficient & 0.213 \\
Cyclic strength coefficient (GPa) & 1.000 \\
Cyclic strain hardening exponent & 0.200 \\
\hline
\end{tabular}

thickness of $0.7 \mathrm{~mm}$ were machined as shown in Fig. 3. Stress-strain reaction of cyclic loading materials is exclusive in relation to lower monotonic loading. For fatigue analysis, very important to study the cyclic material conduct for strength and life investigation. Fatigue itself is a procedure of Initial Crack Length (ICL) and Fatigue Crack (FC) because of cyclic loading. The low material cyclic fatigue properties of AISI 302 have been recorded in (Table 2). All of these properties referred on the Table $1-3$, respectively, among the data must be provided to the software to generate the crack mesh.

Fractographic analysis: Fracture surface observations carried out to identify the mechanism of tensile test fracture of stainless steel AISI 302. Three specimens with thickness $0.7 \mathrm{~mm}$ have been used. There presentative Scanning Electron Microscope (SEM) fractography of the tensile fractured specimens with $10000 \mathrm{X}$ observation shows, the tensile test represented by Fig. 4. Through observation, tensile fracture occurs mostly intergranular (typically dimple) fracture mode. That could be recognized easily in $300 \mu$ sample at higher magnification as compared to $100 \mu$ sample because the dimples are the concentration stresses area.The discussions focused on the differences on the surface of the specimen due to the effect of specimen micro structure and the bulk material properties. The significant difference was seen the pattern, the cleavage and dimples in the fracture surface.

\section{RESULTS AND DISCUSSION}

Process for anticipating life with VAL becomes of exceptionally and complex if one purposes for aprecis evaluation. Summary of structures associated through the $\mathrm{VAL}$ in the prototypes, for example, similar to cooperation (acceleration and retardation), plastic zone development and $\mathrm{CC}$ create the expectation very accurate, however, on the cost of multifaceted nature and muddled calculations. Moreover, the extent of these influences relies upon loading factors, example material properties, geometry, microstructure and condition. The utilization of scientific or mathematical models to reenact break development lowers than arbitrary loading is vital to lessen the quantity of experiments necessary for any weariness FCG investigation. The FCG rates are different for different ICL, while the curves have a similar performance. Figure 5 demonstrate the relations between initial crack lengthICL (a $=0.6 \mathrm{~mm})$ and $(\mathrm{N}=1132$ kilocycles) determine that the greater estimations of ICL drive to the reduced number of cycles for crack growth CG. For an assumed stress variety, the numbers of cycle'srequisite for $\mathrm{CG}$ is determined on the ICL. The stressambition extend in front of the Crack Tip (CT) will be higher for a more profound crack because the Stress Intensity Factor (SIF) fora crack depth. It has been practically confirmed that the comparison between long and short crack shows the number of cycles required for CG in structures having a long crack and lessin the structures having a short crack. An important point in this work is to demonstrate the model ability (Walker Model) 
(a)

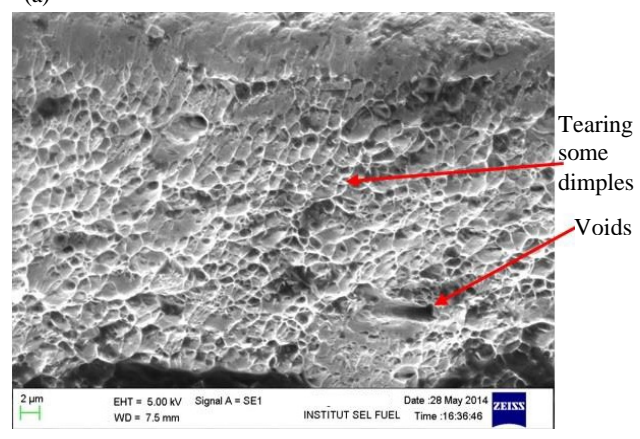

(b)

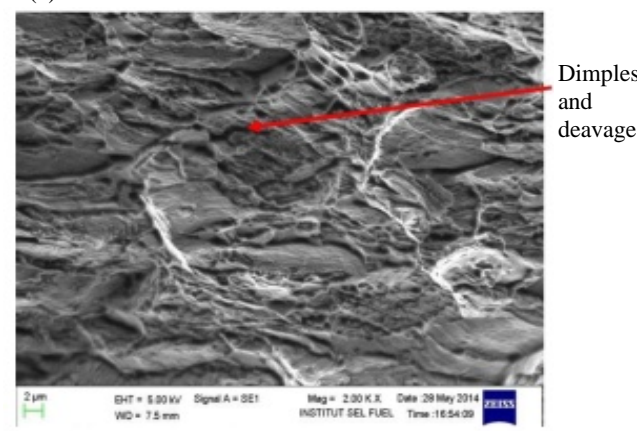

Fig. 5: SEM tensile specimens fracture surface, with a thickness of $0.7 \mathrm{~mm}$ stainless steel $\mathrm{S} 302$; a) Magnification at $100 \mathrm{X}$ and b) Magnification at 2000X

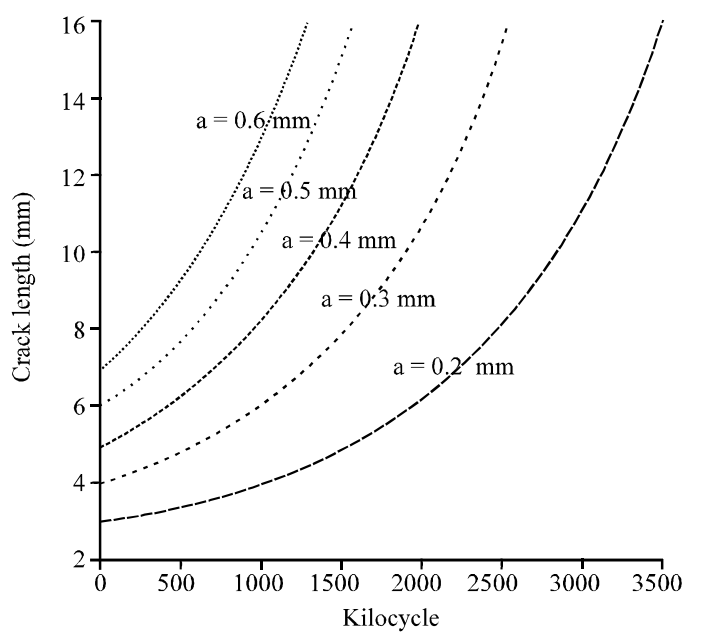

Fig. 6: Thin cylinder a-N curve under different ICL

to recreate the impacts of the ICL on FCG ratio. Figure 6 shows that the FCG ratio is diverse for various ICL while that the curves have a similar behaviour. The curves display that augmentation ICL by $(\mathrm{a}=0.65 \mathrm{~mm}$ ) stimulates to minimize the life by $23 \%$ and this rate rise with little $(\mathrm{a}=0.3,0.4 \mathrm{~mm})$ and less with high estimation of $(\mathrm{a}=0.5,0.6 \mathrm{~mm}$ ). Additional aspect was measured in this study, the impact of dissimilar rate of variable load which

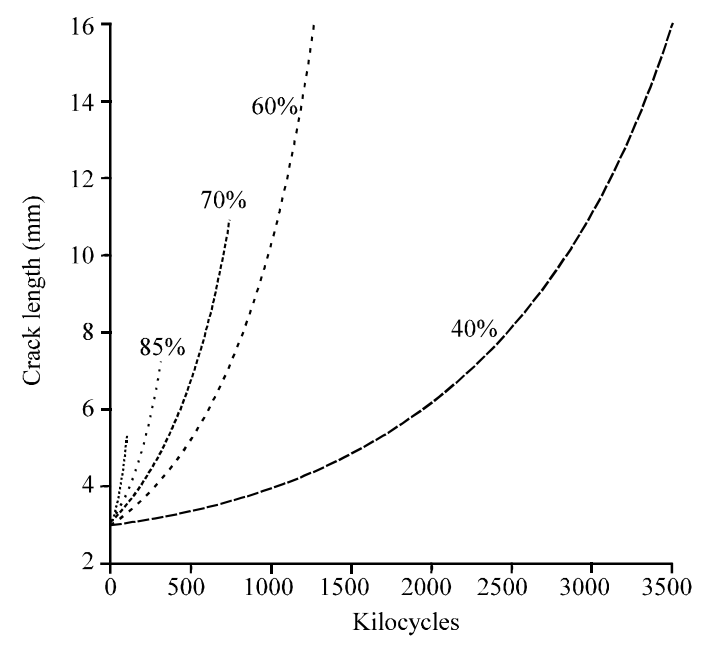

Fig. 7: Thin cylinder a-N curve under different VAL

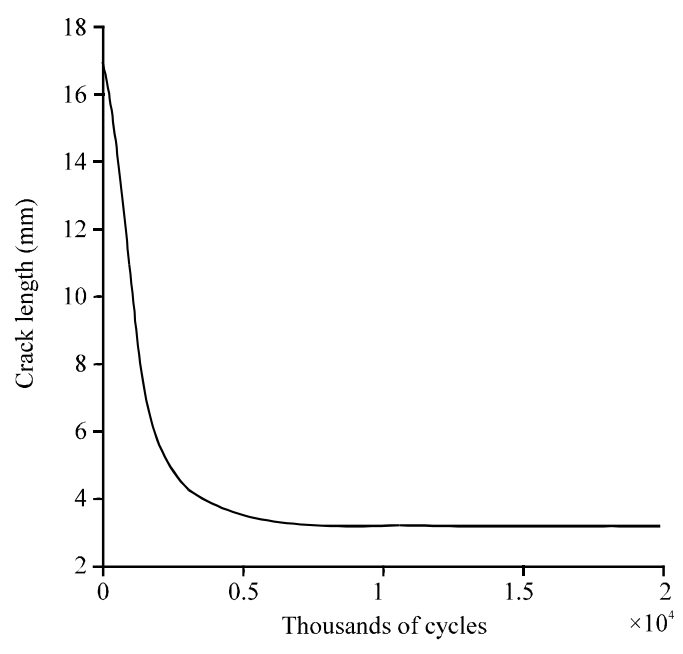

Fig. 8: Thin cylinder fatigue life curve with ICL of using walker FCG Model

demonstrates the impact of utilizing full load at that point $(40,60,70$ and $85 \%)$ of the full variable load. Higher estimation of ICL when the load increases the number of cycle decrees with addition limiting the FL. The Fatigue Life (FL) gives a less life for longer initial crack length (ICL). Figure 7 shows that limiting the variable load growth the FL of the model geometry. The life for full load is $1.07 \mathrm{E} 05$ cycles, however, for $40 \%$ of same load is $3.11 \mathrm{E} 06$ cycles. These qualities can be available in another way, which demonstrates the load life curve for load rate. The angle parametric consumes greatly influence of full load FL, this is demonstrates obviously in Fig. 8 and 9. Such as this angle growth prompts less life. For $30^{\circ}$ the life angle parametric is $1.13 \mathrm{E} 07$ cycles but for $90^{\circ}$ angle is $3.11 \mathrm{E} 06$. 


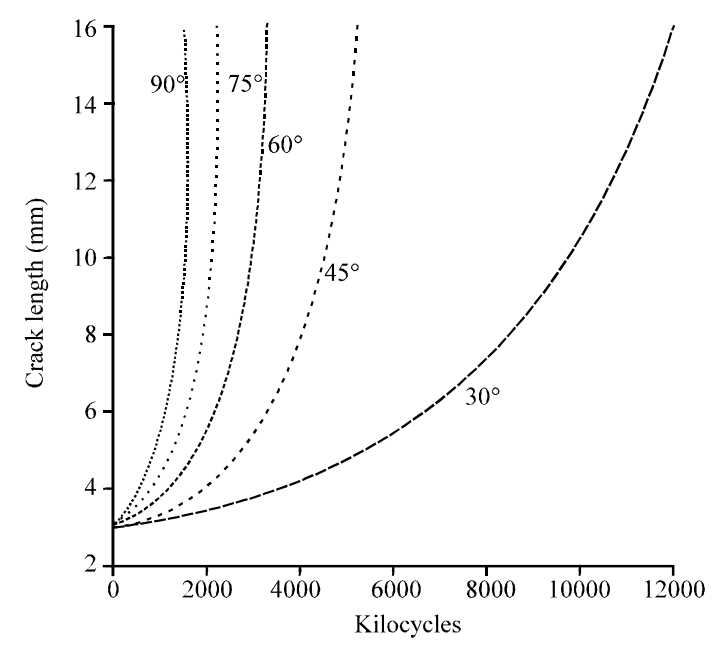

Fig. 9: Thin cylinder a-N curve under different parametric angle for ICL $0.2 \mathrm{~mm}$

\section{CONCLUSION}

A FCG of thin walled pipe with surface crack under VA loading was studied and showed the behavior of FCG with Walker Model used Zencrack Software discovers the influence of variables influencing FCG rate, for example, parametric angle, crack length and load percentage. Through the current progress in calculation innovation, several Ffanalyses are attempting to simulate crack propagation.

A model of FCG for surface under variable loading were achieved the imitation gave a decent figure for FCG rate for various ICL under these sorts of loadings in cylinders. Higher estimation of ICL when the load increases the number of cycle decrees with addition limiting the FL. The parametric angle here a high ruffle on fatigue life, i.e. with addition of this angle the life (number of cycles) will increase. The suggestion model which capable to describing fracture region (3) of FCG curve includes the R-ratio effects. For furthermore, think about requirements to consider alternate factors, for example, other FCG Models, stochastic finite element and approval with experimental research.

\section{REFERENCES}

Alaoui, A.E.M., D. Thevenet and A. Zeghloul, 2009. Short surface fatigue cracks growth under constant and variable amplitude loading. Eng. Fract. Mech., 76: 2359-2370.

Bian, L.C. and J.K. Lim, 2003. Fatigue life prediction of the plates with an inclined surface crack. Intl. J. Fatigue, 25: 521-531.

Chen, Y.Z. and X.Y. Lin, 2008. Elastic analysis for thick cylinders and spherical pressure vessels made of functionally graded materials. Comput. Mater. Sci., 44: $581-587$.

Ghidini, T. and C.D. Donne, 2009. Fatigue life predictions using fracture mechanics methods. Eng. Fract. Mech., 76: 134-148.

Huang, X., M. Torgeir and W. Cui, 2008. An engineering model of fatigue crack growth under variable amplitude loading. Intl. J. Fatigue, 30: 2-10.

Kocanda, D. and M. Jasztal, 2012. Probabilistic predicting the fatigue crack growth under variable amplitude loading. Intl. J. Fatigue, 39: 68-74.

Remes, H., J. Romanoff, I. Lillemae, D. Frank and S. Liinalampi et al., 2017. Factors affecting the fatigue strength of thin-plates in large structures. Intl. J. Fatigue, 101: 397-407.

Siqueira, A.F., C.A.R.P. Baptista, O.L.C. Guimaraes and C.O.F.T. Ruckert, 2010. Describing the total fatigue crack growth curves for aluminum alloys with an exponential equation. Procedia Eng., 2: 1905-1914.

Stephens, R.I., A. Fatemi, R.R. Stephens and H.O. Fuchs, 2000. Metal Fatigue in Engineerring. 2nd Edn., John Wiley \& Sons, Hoboken, New Jersey, USA., Pages: 473. 